\title{
COMPARATIVE ANALYSIS OF MODELS OF DYNAMIC WELDING ARC ${ }^{*}$
}

\author{
I.V. PENTEGOV and V.N. SYDORETS \\ E.O. Paton Electric Welding Institute, NASU \\ 11 Bozhenko Str., 03680, Kiev, Ukraine. E-mail: office@paton.kiev.ua
}

Keywords: gas-shielded arc welding using consumable and non-consumable electrode, dynamic arc, mathematical model, power balance, investigations

There are number of dynamic arc models at present time. Therefore, the researchers when solving the specific tasks on investigation of processes in power source-welding arc system face with the problem of selection of a model providing complete description of the main arc peculiarities in each specific case. This paper is dedicated to objective estimation and comparison of models of dynamic arc and elaboration of recommendations for model selection on this basis.

Some progress in description of welding arc of constant length (non-consumable electrode) as part of electric circuit was achieved using mathematical model of dynamic arc, developed at the E.O. Paton Electric Welding Institute (PWI-MA) [1]. PWI-MA was further developed in works [2, 3]. Arc column in PWI-MA has been phenomenological considered as heat inertial macroobject, for which the following power balance is valid:

$$
\frac{d Q}{d t}=P-P_{\theta},
$$

where $Q$ is the internal energy of arc column; $P$ and $P_{\theta}$ is the input and output power, respectively. All isoenergetic identical states in PWIMA are characterized by one parameter, i.e. arc state current $i_{\theta}[1,3]$. It determines such characteristics of the arc column as static resistance

$$
R_{\mathrm{st}}\left(i_{\theta}\right)=\frac{U_{\mathrm{col}}\left(i_{\theta}\right)}{i_{\theta}}
$$

and output power

$$
P_{\theta}=U_{\text {col }}\left(i_{\theta}\right) i_{\theta},
$$

where $U_{\text {col }}$ is the function defining static voltampere characteristic (SVAC) of the arc column.

Input power is determined by arc column resistance as well as value of its transitional current:

$$
P=R_{\mathrm{st}}\left(i_{\theta}\right) i^{2}=\frac{U_{\mathrm{col}}\left(i_{\theta}\right)}{i_{\theta}} i^{2} .
$$

Voltage on the arc column in dynamic is found from expression

$$
u_{\mathrm{col}}=\frac{U_{\mathrm{col}}\left(i_{\theta}\right)}{i_{\theta}} i .
$$

State current $i_{\theta}$ for any representative point on plane, corresponding to arc column dynamic states in the coordinates of arc column voltage arc current $\left(u_{\mathrm{col}}, i\right)$, is determined as $x$-coordinate of cross point of beam from the origin, passing through this point, with arc column SVAC. Work [3] shows that PWI-MA equation in differential form [2] corresponds to energy balance equation (1):

$$
\theta \frac{d i_{\theta}^{2}}{d t}=i^{2}-i_{\theta}^{2}
$$

which is electroengineering analog of equation (1). At that internal energy of arc column equals [3]

$$
Q=2 \theta \int_{0}^{i_{\theta}} U_{\operatorname{col}}\left(i_{\theta}\right) d i_{\theta},
$$

where $\theta$ is the time constant of arc column.

Since all other models use such a value as arc column conductivity $g$ [4], PWI-MA equation

\footnotetext{
*Translation of paper from «Avtomaticheskaya Svarka» Journal, 1989, No. 2, pp. 33-36. Monitoring of references to the papers, published in this Journal, showed that paper of I.V. Pentegov and V.N. Sydorets is one of the most cited in area of welding arc modelling. It is dedicated to comparison of dynamic model of arc, being developed at the E.O. Paton Electric Welding Institute, with other models and is still of high interest up to the present moment. Unfortunately, «Avtomaticheskaya Svarka» Journal was not translated in English in 1989. However, current reality of the world scientific community necessitates publications in English. Therefore, the Editorial Board of the Journal has made a decision to publish this paper in «The Paton Welding Journal» in order to make it more accessible to wider
} audience of researchers, post-graduate students and students. 
(7) is transferred in conductivity terms using formula (2), from which

$$
g=\frac{i_{\theta}}{U_{\operatorname{col}\left(i_{\theta}\right)}} .
$$

PWI-MA equation is converted in $g$-form by differentiation of expression (8) by time and substituting found $d i_{\theta} / d t$ value in equation (6):

$$
\left[\frac{2 \theta}{1-\frac{g}{G_{\mathrm{dif}}(g)}}\right] \frac{1}{g} \frac{d g}{d t}+1=\frac{i^{2}}{g^{2} U_{\mathrm{col}}^{2}(g)}=\frac{i^{2}}{g P_{\theta}},
$$

where $G_{\mathrm{dif}}=\left(d U_{\mathrm{col}} / d i_{\theta}\right)^{-1}$ is the differential conductivity. For making a comparison let us provide equations of arc column dynamic of all widespread models.

Cassie's model [5]:

$$
\frac{\theta_{\mathrm{C}}}{g} \frac{d g}{d t}+1=\left(\frac{i}{g U_{\mathrm{C}}}\right)^{2}
$$

where $\theta_{\mathrm{C}}$ is the arc time constant in Cassie's model; $U_{\mathrm{C}}$ is the static voltage on the arc column, which is constant in Cassie's model.

Mayr's model [6]:

$$
\frac{\theta_{\mathrm{M}}}{g} \frac{d g}{d t}+1=\frac{i^{2}}{g P_{\mathrm{M}}}
$$

where $\theta_{\mathrm{M}}$ is the time constant in Mayr's model; $P_{\mathrm{M}}$ is the output power, which is constant in Mayr's model.

Zarudi's model [7, 8]:

$$
\frac{\theta \mathrm{z}}{g} \frac{d g}{d t}+1=\frac{i^{2}}{g^{k+1} X_{k}},
$$

where $\theta_{Z}=(1+k) \theta$ is the value, which can be considered as time constant in Zarudi's model; $k$ is the index of plasma non-linearity depending on gas nature, in which arc burns; $X_{k}$ is the constant at fixed $k$.

Schellhase's model [9]:

$$
\frac{\theta_{\mathrm{S}}}{g} \frac{d g}{d t}+1=\frac{1}{g U_{\mathrm{col}}(i)},
$$

where $\theta_{\mathrm{S}}$ is the time constant in Schellhase's model.

Comparison of the models show that the time constants are different in all models. Let us consider what are the conditions when expression

$$
\tau=\frac{2 \theta}{1-\frac{g}{G_{\operatorname{dif}}(g)}}
$$

in equation (9) is the constant.

Obviously, for this it is necessary that

$$
\frac{g}{G_{\mathrm{dif}}(g)}=\frac{i_{\theta}}{U_{\mathrm{col}}\left(i_{\theta}\right)} \frac{d U_{\mathrm{col}}}{d i_{\theta}}=n=\text { const. }
$$

Separating the variables and integrating the latter equation the following is received:

$$
U_{\text {col }}\left(i_{\theta}\right)=U_{0}\left(\frac{i_{\theta}}{I_{0}}\right)^{n},
$$

where $U_{0}$ and $I_{0}$ are the coordinates of one of SVAC fixed points.

Thus, Cassies's, Mayr's and Zarudi's models follow from PWI-MA (9) in a special case, when SVAC has power form with index of power $n$, and equation (9) is written as

$$
\frac{2 \theta}{1-n} \frac{1}{g} \frac{d g}{d t}+1=\left(\frac{I_{0}^{n}}{U_{0}}\right)^{\frac{2}{1-n}} \frac{i^{2}}{g^{\frac{2}{1-n}}} .
$$

Cassie's model (10) is received at $n=0$. Then $U_{\mathrm{C}}=U_{0}$ and $\theta_{\mathrm{C}}=2 \theta$. Mayr's model (11) takes place at $n=-1$. In this case $P_{\mathrm{M}}=U_{0} I_{0}$ and $\theta_{\mathrm{M}}=$ $=\theta$. Zarudi's model (12) is realized at $n=-(1-$ $-k) /(1+k)$. Then

$$
X_{k}=I_{0}^{2}\left(\frac{U_{0}}{I_{0}}\right)^{1+k} \text { and } \theta_{\mathrm{Z}}=(1+k) \theta=\frac{2 \theta}{1-n} .
$$

Schellhase's model (13) can not be received from PWI-MA (9) under any conditions. It can be superposed neither with Cassie's models no with Zarudi's model. Obviously, it is explained by the fact that Schellhase's model [9] was built on concepts, which did not use power balance equation (1).

Specific attention is to be given to interpretation of coefficient $\tau$ from formula (14). It was mentioned above that this coefficient remains constant in the case of SVAC power function (15). Therefore, it is called an arc time constant in different models. In general case $\tau$ value depends on conductivity and can not be interpreted as arc time constant. Moreover, as it is shown in work [3], the transfer processes in the circuit with arc generally can not be characterized by process time constant. This conclusion matches with the results of experimental investigations in works [10, 11]. Thus, PWI-MA eliminates existing contradiction on variability of arc time constant. The arc time constant $\theta$ in PWI-MA is the invariable value, but it is not time constant of the process. And generally coefficient $\tau$ is the variable value. 
It follows from mentioned above that PWIMA applicable to welding arc has series of advantages in comparison with other methods. PWI-MA is the most general of all models. It can use SVAC of any type (including areas with independent and growing SVAC), that is very important in welding arc modelling. PWI-MA, in terms of electric engineering, uses a value of state current, that has apparent advantages in comparison with other models, which use values of conductivity or arc column resistance. PWIMA allows easily determining such important energy parameters of the arc column as output power and internal energy of the arc column. Formula (7) for internal energy of the arc column, obtained with the help of PWI-MA, allows expanding sphere of application of dynamic arc equations, including for the cases of arcs with varying in time arc length $l_{\mathrm{a}}(t)$ as well as moving and blown arcs, that is in principle inaccessible for alternative arc models.

Using equality

$$
U_{\text {col }}\left(i_{\theta}\right)=l_{\mathrm{a}}(t) E_{\text {col }}\left(i_{\theta}\right),
$$

where $E_{\mathrm{col}}\left(i_{\theta}\right)$ is the average value of electric field intensity in the arc column (SVAC of the arc is determined particularly for this value of intensity, in the case of cylindrical arc, $E_{\text {col }}\left(i_{\theta}\right)$ agrees with real electric field intensity in the arc column). Let us transfer equation (7) in the following form:

$$
Q=2 \theta l_{\mathrm{a}}(t) \int_{0}^{i_{\theta}(i)} E_{\mathrm{col}}\left(i_{\theta}\right) d i_{\theta} .
$$

Here out

$$
\begin{gathered}
\frac{d Q}{d t}=\frac{\partial Q}{\partial i_{\theta}} \frac{d i_{\theta}}{d t}+\frac{\partial Q}{\partial l_{\mathrm{a}}} \frac{d l_{\mathrm{a}}}{d t}= \\
=2 \theta l_{\mathrm{a}} E_{\mathrm{col}}\left(i_{\theta}\right) \frac{d t_{\theta}}{d t}+2 \theta \frac{d l_{\mathrm{a}}}{d t} \int_{0}^{i_{\theta}} E_{\mathrm{col}}\left(i_{\theta}\right) d i_{\theta} .
\end{gathered}
$$

Substituting this expression together with (3) and (4) in equation (1), PWI-MA equation for changing arc length is received in general case:

$$
\theta \frac{d i_{\theta}^{2}}{d t}=i^{2}-i_{\theta}^{2}\left[1+\frac{2 \theta v_{1}}{l_{\mathrm{a}}}\left[\frac{\int_{i_{\mathrm{col}}}^{i_{\theta}} E_{\left.i_{\theta}\right) d i_{\theta}}}{E_{\mathrm{col}}\left(i_{\theta}\right) i_{\theta}}\right]\right\} \text {, }
$$

where $v_{1}=d l_{\mathrm{a}} / d t$ is the rate of arc length change.

The latter equation assumes the following form of record for general case

$$
\theta \frac{d i_{\theta}^{2}}{d t}=i^{2}-i_{\theta}^{2}\left(1+\frac{v_{1}}{l_{\mathrm{a}}} \frac{Q}{P_{\theta}}\right)
$$

and for particular case of power approximation of SVAC curve (15)

$$
\theta \frac{d i_{\theta}^{2}}{d t}=i^{2}-i_{\theta}^{2}\left(1+\frac{2 \theta v_{1}}{(1+n) l_{\mathrm{a}}}\right) .
$$

In the latter formula $n \neq 1$ since SVAC can not be hyperbola at all integration interval [3] due to inversion into infinity of value of arc internal energy $Q$. Generally, SVAC is not approximated by power function, and it is necessary to use common equation (19). Expression in square brackets is easily calculated at any form of arc SVAC.

Obtained equation reveals more perspectives in solving the problems relating with consumable electrode welding, since this method of welding is the most widespread.

Gas blowing of welding arc provides for additional mechanism of energy output, power of which at longitudinal blowing

$$
P_{v}=\frac{V_{\mathrm{sp}}}{V_{\mathrm{a}}} Q=\frac{\left|v_{\mathrm{bl}}\right|}{l_{\mathrm{a}}} Q,
$$

at transverse blowing

$$
P_{v}=\frac{V_{\mathrm{sp}}}{V_{\mathrm{a}}} Q=\frac{2 l_{\mathrm{e}}\left|v_{\mathrm{bl}}\right|}{\pi l_{\mathrm{a}} r_{\mathrm{a}}} Q,
$$

where $V_{\mathrm{sp}}$ is the volume of plasma being blown per unit of time.

For longitudinal blowing

$$
V_{\mathrm{sp}}=\pi r_{\mathrm{a}}^{2}\left|v_{\mathrm{bl}}\right|,
$$

for transverse blowing

$$
V_{\mathrm{sp}}=2 r_{\mathrm{a}} l_{\mathrm{e}}\left|v_{\mathrm{bl}}\right| .
$$

Here $v_{\mathrm{bl}}$ is the blowing rate; $r_{\mathrm{a}}$ is the arc column radius; $l_{\mathrm{e}}$ is the distance between the electrodes, in general case $l_{\mathrm{e}} \leq l_{\mathrm{a}} ; V_{\mathrm{a}}$ is the arc volume.

Equation of power balance in this case has a form of

$$
\frac{d Q}{d t}=P-P_{\theta}-P_{v}
$$

PWI-MA equation for longitudinal blow is

$$
\theta \frac{d i_{\theta}^{2}}{d t}=i^{2}-i_{\theta}^{2}\left(1+\frac{\left|v_{\mathrm{bl}}\right|}{l_{\mathrm{a}}} \frac{Q}{P_{\theta}}\right),
$$

and for transverse blow

$$
\theta \frac{d i_{\theta}^{2}}{d t}=i^{2}-i_{\theta}^{2}\left(1+\frac{2}{\pi} \frac{l_{\mathrm{e}}}{l_{\mathrm{a}}} \frac{\left|v_{\mathrm{bl}}\right|}{r_{\mathrm{a}}} \frac{Q}{P_{\theta}}\right) .
$$


Equation (25) is expedient to be used for description of processes with plasma arc application, when longitudinal gas blow is inseparable part of the process. Equation (26) shall be used in transverse blowing as well as for different cases of arc movement, including arc movement in magnetic field, at that $v_{\mathrm{bl}}$ is considered as arc movement rate. It should be noted that different combinations of equations (19), (24) and (25) are possible. For example PWI-MA equation

$$
\theta \frac{d i_{\theta}^{2}}{d t}=i^{2}-i_{\theta}^{2}\left(1+\frac{\left|v_{\mathrm{bl}}\right|+v_{1}}{l_{\mathrm{a}}} \frac{Q}{P_{\theta}}\right)
$$

is valid in arc length change and longitudinal arc blow.

\section{Conclusions}

1. Mathematical model of dynamic arc is valid for any types of SVAC and allows solving the problems in the electroengineering terms. It can be used in the cases of varying length arcs (including the consumable electrode arc), moving arcs and gas blown arcs, and provides the method for determination of internal energy and output power, that is impossible in alternative models.

2 . Obtained results are valid not only for welding, but for plasma arcs and rupturing arcs in electric apparatuses.

3. Using the computer PWI-MA equations allows investigating evolutionary processes in power source-arc system for various cases of gas- shielded welding, namely consumable-electrode in inert gases and mixture of active gases and tungsten-electrode in inert gases welding.

1. Pentegov, I.V. (1976) Mathematical model of column of electric dynamic arc. Avtomatich. Svarka, 6, $8-12$

2. Pentegov, I.V., Sydorets, V.N., Genis, I.A. (1984) Problems of modelling of welding arc dynamics as part of electric circuit. Ibid., 10, 18-23.

3. Pentegov, I.V., Sydorets, V.N. (1988) Energy parameters in mathematical model of dynamic welding arc. Ibid., 11, 36-40."

4. Novikov, O.Ya. (1987) Electric arc stability. Leningrad: Energiya.

5. Cassie, A.M. (1939) A new theory of arc rupture and circuit severity. CIGRE, 102, 1-10.

6. Mayr, O. (1943) Beitrage zur Theorie des statischen und des dynamischen Lichtbogens. Arch. fuer Elektr., 38, 588-608.

7. Zarudi, M.E. (1971) On effect of non-linear properties of plasma on character of non-stationary processes in column of channel arc (Problems of theory and calculation). Zhurnal Tekhn. Fiziki, 41(4), 734743 .

8. Zarudi, M.E. (1977) Criteria of existence and stability of stationary modes in alternating current inductive circuit with arc. Elektrichestvo, 4, 53-60.

9. Schellhase, M. (1971) Mathematical model of transfer processes in welding arc and its investigations. Avtomatich. Svarka, 7, 13-16.

10. Kruchinin, A.M., Peshekhonov, V.I., Lazutkin, Yu.V. et. al. (1983) Calculation of arc time constant on computer. In: Mathematical modelling and disign of electric arc and plasma steel furnaces: Transact. of VNIIETO, 46-49. Moscow: Energoatomizdat.

11. (1981) Current interruption in high-voltage mains. Moscow: Energoizdat.

Received 04.11.2015

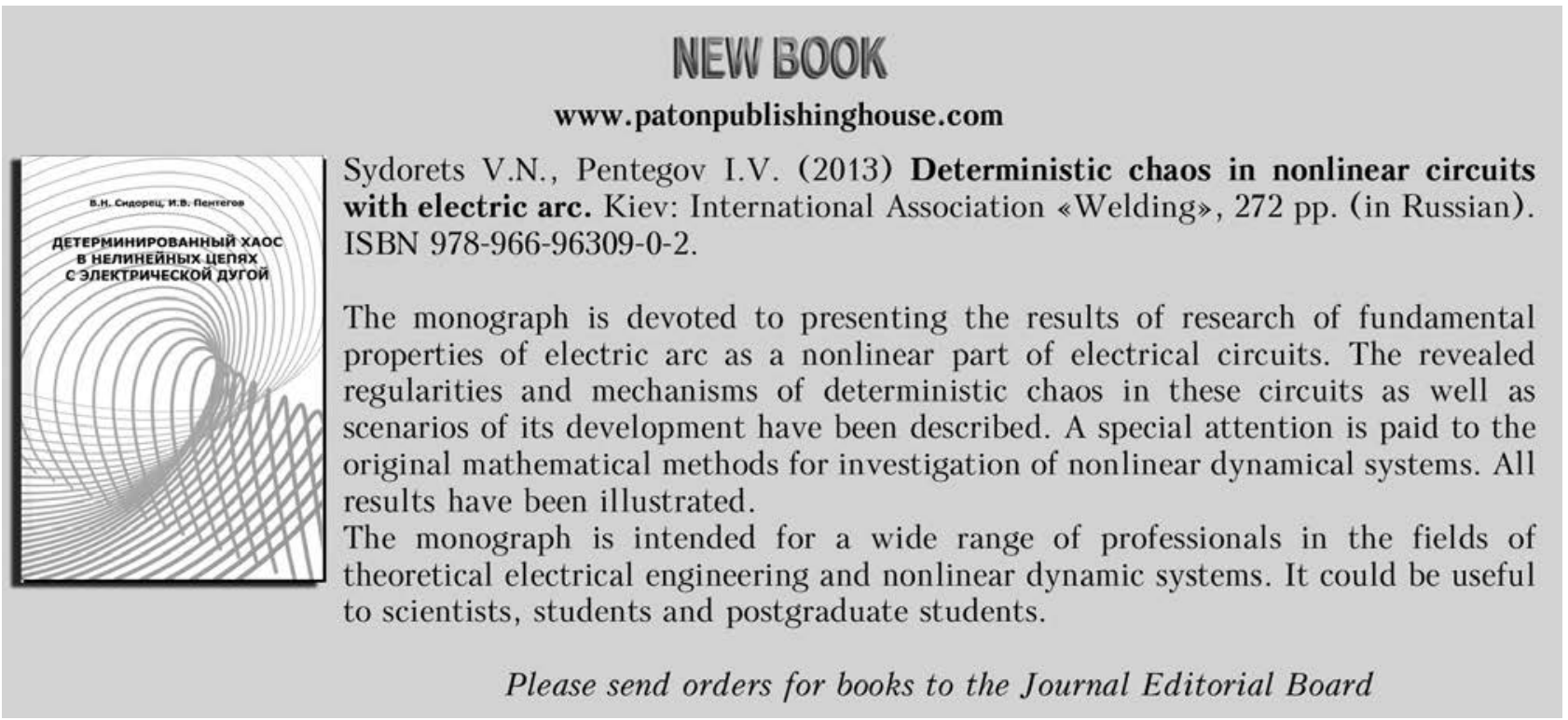

*Pentegov, I.V., Sidorets, V.N. (1990) Energy parameters in a mathematical model of a dynamic welding arc. Welding Int., 4 (4), $272-275$. 